\title{
Atomic density and temperature distributions in magneto-optical traps
}

\author{
A. S. Arnold* and P. J. Manson \\ Department of Physics, University of Otago, P.O. Box 56, Dunedin, New Zealand
}

Received July 6, 1999; revised manuscript received November 23, 1999

\begin{abstract}
A theoretical investigation into density, pressure, and temperature distributions in magneto-optical traps is presented. After a brief overview of the forces that arise from reradiation and absorption, a condition that the absorptive force be conservative is used to show that, if the temperature is uniform throughout the trap, any density solutions to the force equations will not be physical. Further, consistent density solutions are unlikely to exist at all. In contrast, with a varying temperature reasonable solutions are demonstrated, with some restrictions. Doppler forces involved in ring-shaped trap structures are used to calculate orbit radii in racetrack geometry traps, and corrections to the present discrepancy between theoretical and experimental studies are discussed in the context of reradiation and diffusion. (c) 2000 Optical Society of America [S0740-3224(00)01104-8]
\end{abstract}

OCIS codes: $020.7010,140.3320$

\section{INTRODUCTION}

The realization of the magneto-optical trap (MOT) in $1987^{1}$ created a useful and reliable source of cold atoms for many applications, including atom interferometry, atomic fountain clocks, and nonlinear optics experiments. These traps are now commonly loaded from an atomic vapor $^{2}$ and have been used to attain temperatures below the Doppler limit. ${ }^{3-7}$ The density and temperature in a MOT have been under scrutiny lately in attempts to reach higher phase space densities, facilitating the production of Bose-Einstein condensates in neutral atomic gases. $^{8-11}$

There have been several experimental and theoretical studies of the density distribution in MOT's. In the simplest case of exactly counterpropagating trapping laser beams, the trapped cloud is ellipsoidal. With a small number of trapped atoms the atoms are essentially noninteracting, and the density distribution is expected to be Gaussian with a radius that remains constant as more trapped atoms are added to the MOT. ${ }^{10}$ In this temperature-limited (TL) regime the density increases linearly with the number of trapped atoms. As the number of trapped atoms increases, scattering of the trapping light by atoms within the trap introduces an interatomic repulsive force. In this multiple-scattering regime the density distribution is either approximately uniform ${ }^{12}$ or Gaussian, ${ }^{10}$ and in both cases the density is constrained below a maximum value that is independent of the number of trapped atoms.

In addition to the standard symmetric trap geometry, if the trapping laser beams are aligned in a racetrack geometry complicated structures including rings, double rings, and rings with central cores can be observed. ${ }^{12-17}$ These systems are of interest because analysis of features such as the conditions for formation of the different types of structure and the radii of the rings formed can be used to verify models of the processes creating forces in the trap.

We believe that approximations made in some theoret- ical treatments of both of these geometries have either hidden some important features or distorted the conclusions. Here we aim to rework some aspects of the theory, avoiding some of the approximations and showing how the conclusions must be modified.

We start in Section 2 with the simpler geometry of retroreflected trapping beams. After briefly outlining the origin of the forces acting on the atoms in the MOT, we show in Subsection 2.B that physically reasonable density distributions in a MOT cannot be found if the temperature is assumed to be uniform. It is further argued that solutions to the density equations may not exist at all. In contrast, if the temperature is allowed to vary we find, in Subsection 2.C, physically reasonable density, pressure, and temperature distributions. Although we could calculate these distributions only by omitting the reradiation force, we believe that physical solutions would still exist after its inclusion. In Subsection 2.D, by making an approximation to the intensity imbalance force, we discuss and compare relatively simple spherically symmetric density distributions that exist under both varying- and constant-temperature conditions. Then in Section 3 we examine the more complicated racetrack trapping beam geometry. By considering the centripetal and tangential forces produced by the beams in a simple one-dimensional (1D) model, we find in Subsection 3.A the radii of stable orbits. These results are compared with previous $1 \mathrm{D}$ calculations and with a three-dimensional (3D) Monte Carlo simulation. We show that the approximations used in the past cause significant distortions to the conclusions that can be drawn.

\section{RERADIATION AND ABSORPTION}

A. Forces on the Atoms

Following previous authors, ${ }^{12}$ we focus on three of the position-dependent forces that act on the atoms in a MOT, i.e., the restoring force produced by the trapping lasers, 
the force caused by absorption of the trapping lasers by atoms in the trap, and the force that is due to photons reradiated by other atoms in the cloud after absorption. The model permits the assumption of either a Doppler or a sub-Doppler average temperature, with the combination of the applied forces and the thermal motion of the atoms determining the size and nature of the atomic density distribution.

Throughout the analysis, unless otherwise stated, all laser beam intensities are assumed to be equal in the MOT. This should lead to an isotropic temperature among the MOT atoms and also to a trapping force ${ }^{18}$ of the form $\mathbf{F}_{\text {spring }}=-k_{\text {spring }}(x, y, 2 z)$, where $k_{\text {spring }}$ is a constant. ${ }^{19}$ The force $\mathbf{F}_{\text {spring }}$ is conservative (i.e., $\boldsymbol{\nabla}$ $\times \mathbf{F}_{\text {spring }}=\mathbf{0}$ ). The spring constant $k_{\text {spring }}$ of the MOT can be obtained by either Doppler or sub-Doppler calculations or experiment ${ }^{10}$; thus the following theory may be tailored to both situations as required. A recent paper ${ }^{20}$ has shown how scattered radiation affects the subDoppler cooling mechanisms in a MOT. The sub-Doppler enhancement of the spring constant in the trap will be similarly affected. Such effects are omitted here but might be treated in a similar method to that of Ref. 20 .

We now consider the two other position-dependent forces that are present in a MOT. The first is the force that is due to changes in the laser beam intensities when light is absorbed by the MOT atoms. ${ }^{21}$ The second is a force that is due to the reradiation of the absorbed laser light. ${ }^{12}$ Both forces were considered in detail by Sesko et al. in Ref. 12 , and the notation used here will be similar.

The intensity $I_{x \pm}(\mathbf{r})$ of a collimated laser beam propagating in the $\pm x$ direction through a cloud of atoms is governed by the partial differential equation $\partial I_{x^{ \pm}}(\mathbf{r}) / \partial x$ $=\mp \sigma_{L} I_{x^{ \pm}}(\mathbf{r}) n(\mathbf{r})$, where $n(\mathbf{r})$ is the atomic number density and $\sigma_{L}$ is the cross section for absorption of laser photons. This equation can be solved to yield

$$
I_{x^{ \pm}}(\mathbf{r})=I_{x_{\infty}} \exp \left[\mp \sigma_{L} \int_{\mp \infty}^{x} n(u, y, z) \mathrm{d} u\right],
$$

where $I_{x_{\infty}}$ is the unattenuated beam intensity.

The absorption force $\mathbf{F}_{\text {abs }}$ on an atom that is due to the resultant intensity imbalance between the two $\pm x$ laser beams consists solely of an $x$ component $^{12}$ :

$$
F_{\mathrm{abs}_{x}}=-2 k_{\mathrm{abs}} I_{x_{\infty}} \int_{0}^{x} n(u, y, z) \mathrm{d} u,
$$

where $k_{\mathrm{abs}}=\sigma_{L}{ }^{2} / c$ and the absorption is assumed small enough to permit a linear approximation to the exponential in Eq. (1). The symmetry of the spring constant and beam intensities implies that the MOT number density will be even in $x$. Similar force components arise from the MOT laser beams in the $y$ and $z$ directions with unattenuated intensities $I_{y_{\infty}}$ and $I_{z_{\infty}}$, respectively. The divergence $\boldsymbol{\nabla} \cdot \mathbf{F}_{\mathrm{abs}}=-k_{\mathrm{abs}} I_{\mathrm{tot}} n(\mathbf{r})$, where $I_{\text {tot }}=2\left(I_{x_{\infty}}+I_{y_{\infty}}\right.$ $+I_{z_{\infty}}$ ), is always negative; hence the absorption force is compressive. For most density distributions $\boldsymbol{\nabla} \times \mathbf{F}_{\mathrm{abs}}$ $\neq \mathbf{0}$, and the absorption force is not conservative.

The other force on atoms in a MOT is the reradiation force $\mathbf{F}_{\text {rerad }}$. The atoms are continually reemitting photons absorbed from the laser beams, and this reemitted light can be absorbed by other atoms in the cloud, producing a $1 / r^{2}$-dependent repulsive force between pairs of atoms with separation $r$. Because the frequency distribution of the reradiated photons may be different from that of the incident laser photons, the probabilities of an atom's absorbing a scattered photon and a laser photon are slightly different, so the cross section $\sigma_{R}$ for the reradiated light is distinct from $\sigma_{L}$. The spatial distribution of spontaneous emission is assumed to be isotropic.

The reradiation force on an atom at a point $\mathbf{r}$ in an atomic distribution is given by ${ }^{12}$

$$
\mathbf{F}_{\text {rerad }}(\mathbf{r})=\frac{k_{\text {rerad }}}{4 \pi} \int n\left(\mathbf{r}^{\prime}\right) I\left(\mathbf{r}^{\prime}\right) \frac{\mathbf{r}-\mathbf{r}^{\prime}}{\left|\mathbf{r}-\mathbf{r}^{\prime}\right|^{3}} \mathrm{~d}^{3} r^{\prime},
$$

where $k_{\text {rerad }}=\sigma_{R} \sigma_{L} / c$ and it has been assumed that an incident photon is unlikely to be absorbed and emitted more than twice by atoms in the MOT.

It can be shown that $\boldsymbol{\nabla} \cdot \mathbf{F}_{\text {rerad }}=k_{\text {rerad }} I_{\text {tot }} n(\mathbf{r})$ by invoking our earlier assumption that the absorption in the MOT is small. The form of the divergence of $\mathbf{F}_{\text {rerad }}$ is similar to that of $\mathbf{F}_{\text {abs }}$ but with opposite sign so it acts to expand the atomic cloud. It can also be shown by applying Leibnitz's rule that $\mathbf{F}_{\text {rerad }}$ is conservative for all density distributions. If the atomic density distribution is spherically symmetric, then an analog of Gauss's law shows that $\mathbf{F}_{\text {rerad }}$ is radially outward with magnitude

$$
F_{\text {rerad }}(r)=k_{\text {rerad }} I_{\text {tot }} \frac{\int_{0}^{r} n(u) u^{2} \mathrm{~d} u}{r^{2}} .
$$

The three forces discussed above will now be combined to form the total force acting on the atoms in a MOT: $\mathbf{F}_{\text {tot }}=\mathbf{F}_{\text {spring }}+\mathbf{F}_{\text {abs }}+\mathbf{F}_{\text {rerad }}$. This is called the WalkerSesko-Wieman (WSW) model by Ellinger et al. ${ }^{22}$ who provide a more-recent and -precise derivation of the spatially dependent forces in the model. Their derivation reduces to the WSW model at first order.

\section{B. Constant-Temperature Density Distribution}

For any isotropic material with a stress tensor composed solely of normal stresses $P$, the equation $\nabla P(\mathbf{r})$ $=\mathbf{F}_{\text {tot }}(\mathbf{r}) n(\mathbf{r})$ is valid and will therefore hold for the atomic vapor in a MOT. With long-range interactions between atoms in the trap included in the positiondependent pressure, the ideal gas equation may be used to relate the pressure $P$ to the density and temperature at each point in the trapped cloud, ${ }^{12}$ giving

$$
\boldsymbol{\nabla} P(\mathbf{r})=k_{B}[T(\mathbf{r}) \nabla n(\mathbf{r})+n(\mathbf{r}) \nabla T(\mathbf{r})]=\mathbf{F}_{\text {tot }} n(\mathbf{r}) .
$$

At this point, following Ref. 12, the temperature will be assumed constant throughout the distribution. With this restriction, Eq. (5) reduces to

$$
k_{B} T \nabla[\ln n(\mathbf{r})]=\mathbf{F}_{\text {spring }}+\mathbf{F}_{\mathrm{abs}}+\mathbf{F}_{\text {rerad }}=\mathbf{F}_{\text {tot }} .
$$

If $\mathbf{F}_{\text {spring }}$ is the only force considered in Eq. (6), then $n(\mathbf{r})=n_{0} \exp \left[-k_{\text {spring }} r^{2} /\left(2 k_{B} T\right)\right]$. It is also possible to find spherically symmetric solutions to Eq. (6) if the trapping force is simplified to $\mathbf{F}_{\text {spring }}=-k_{\text {spring }} \mathbf{r}$ and only the conservative forces $\left(\mathbf{F}_{\text {spring }}\right.$ and $\left.\mathbf{F}_{\text {rerad }}\right)$ are considered. In 
contrast, we show below that no physically reasonable solution is possible when the nonconservative force $\mathbf{F}_{\mathrm{abs}}$ is included.

The divergence of Eq. (6) can be taken to yield

$$
\begin{aligned}
k_{B} T \nabla^{2}[\ln n(\mathbf{r})] & =\boldsymbol{\nabla} \cdot \mathbf{F}_{\text {tot }} \\
& =\left(k_{\text {rerad }}-k_{\text {abs }}\right) I_{\text {tot }} n(\mathbf{r})-3 k_{\text {spring }},
\end{aligned}
$$

which can be solved for $n .^{12,23}$ However, it should be stressed that a solution to Eq. (7) is not necessarily a solution to Eq. (6). New solutions arise when an equation is differentiated: It is possible to obtain solutions (e.g., the spherically symmetric solutions mentioned above) to Eq. (7) that are not solutions to the original equation (6). In addition, the set of solutions to Eq. (6) is equivalent to a subset within the intersection of the solution spaces of Eq. (7) and the Smoluchowski equation used in Refs. 21 and 22 .

It will now be shown that, unlike for Eq. (7), physical solutions to Eq. (6) do not exist on inclusion of $\mathbf{F}_{\mathrm{abs}}$. A physical density solution is considered here to be a function that tends to zero as the distance from the center of the MOT increases. Our initial analysis requires the density distribution to have a Taylor expansion with a radius of convergence that is large compared with the size of the trapped atom cloud.

As the left-hand side of Eq. (6) is the gradient of a single function, the curl of the right-hand side must be zero, and the condition $\boldsymbol{\nabla} \times \mathbf{F}_{\mathrm{abs}}=\mathbf{0}$ is obtained. Thus Eq. (6) can be solved only when the density distribution is constrained in such a way as to make $\mathbf{F}_{\text {abs }}$ conservative. Although a density distribution that generates a conservative absorptive force provides a possible solution to Eq. (6), some distributions that satisfy the conservative condition on $\mathbf{F}_{\text {abs }}$ will not satisfy Eq. (6). Thus, proving that all density distributions that produce a conservative absorption force are unphysical is actually a stronger condition than is necessary to show that there are no physical solutions to Eq. (6).

Setting the $z$ component of the curl of $\mathbf{F}_{\mathrm{abs}}$ to zero gives

$$
I_{x_{\infty}} \frac{\partial}{\partial y} \int_{0}^{x} n(u, y, z) \mathrm{d} u=I_{y_{\infty}} \frac{\partial}{\partial x} \int_{0}^{y} n(x, u, z) \mathrm{d} u .
$$

We use a Taylor expansion for $n(x, y)$ in the $z=0$ plane, as this is enough to establish the absence of a physical solution. Because of the symmetry of the laser beam and magnetic field arrangement, the density will be even in $x$ and $y$, so the solutions are developed in the positive quadrant only. The Taylor series in a quadrant can be rearranged, assuming absolute convergence, to $n(x, y)$ $=\sum_{N=0}^{\infty} \sum_{j=0}^{N} c_{N-j, j} x^{N-j} y^{j}$. Substituting this equation into the condition for a conservative $\mathbf{F}_{\text {abs }}$ [Eqs. (8)] gives

$$
\begin{aligned}
& \sum_{N=0}^{\infty} \sum_{j=0}^{N} c_{N-j, j} \frac{j}{N-j+1} x^{N-j+1} y^{j-1} \\
& \quad=\mathcal{I} \sum_{N=0}^{\infty} \sum_{j=0}^{N} c_{N-j, j} \frac{N-j}{j+1} x^{N-j-1} y^{j+1},
\end{aligned}
$$

where $\mathcal{I}=I_{y_{\infty}} / I_{x_{\infty}}$ is the ratio of the incident intensities. By transforming the summation indices it can be shown that only the Taylor coefficients with indices that are both even are nonzero.

A recurrence relation for the even series coefficients can be derived, where $\left(\begin{array}{l}i \\ j\end{array}\right)=i ! / j !(i-j)$ !:

$$
c_{2(l-k), 2 k}=\mathcal{I}^{k}\left(\begin{array}{l}
2 l \\
2 k
\end{array}\right) c_{2 l, 0} .
$$

Thus all coefficients of order $N=2 l$ are determined by $c_{N, 0}$, and the entire solution in the $(x, y)$ plane is determined by specifying the form of $n$ on the $x$ (or $y$ ) axis. All density distributions that satisfy the conservative $\mathbf{F}_{\mathrm{abs}}$ condition are therefore of the form

$$
n(x, y)=\sum_{l=0}^{\infty} \sum_{k=0}^{l} \mathcal{I}^{k}\left(\begin{array}{l}
2 l \\
2 k
\end{array}\right) c_{2 l, 0} x^{2(l-k)} y^{2 k}
$$

As noted above, a physically reasonable density distribution for the MOT must be localized in space. However, the distribution in Eq. (11) does not satisfy this condition. Consider, for example, the distribution along the line $y$ $=x / \sqrt{\mathcal{I}}$. Using $\sum_{k=0}^{l}\left(\begin{array}{c}2 l \\ 2 k\end{array}\right)=2^{2 l-1}$ (where $l \geqslant 1$ ), we have

$$
\begin{aligned}
n(x, x / \sqrt{\mathcal{I}}) & =c_{0,0}+\frac{1}{2} \sum_{l=1}^{\infty} c_{2 l, 0}(2 x)^{2 l} \\
& =1 / 2 n(0,0)+1 / 2 n(2 x, 0) .
\end{aligned}
$$

The reason why $n$ is unphysical now becomes clear. Because $n$ must tend to zero along the $x$ axis, Eq. (12) implies that it tends to a nonzero value, $1 / 2 n(0,0)$, along the line $y=x / \sqrt{\mathcal{I}}$. Figure 1 shows a typical density distribution derived in this way. Solutions with $n(0,0)=0$, i.e., a hole in the atomic distribution at the origin, approach zero along both the $x$ and the $y=x / \sqrt{\mathcal{I}}$ axes; however, other paths in the $x y$ plane can be found along which the limiting density is nonzero. Thus all density distributions that satisfy $\boldsymbol{\nabla} \times \mathbf{F}_{\text {abs }}=\mathbf{0}$ (i.e., that generate a conservative $\mathbf{F}_{\mathrm{abs}}$ ) are unphysical, so we must conclude that there are no physical density solutions to Eq. (6).

We have shown that all density distributions constrained to generate a conservative $\mathbf{F}_{\text {abs }}$ are unphysical, but there is also evidence that solutions to Eq. (6) may not exist at all. The density solution series [Eq. (11)] has not

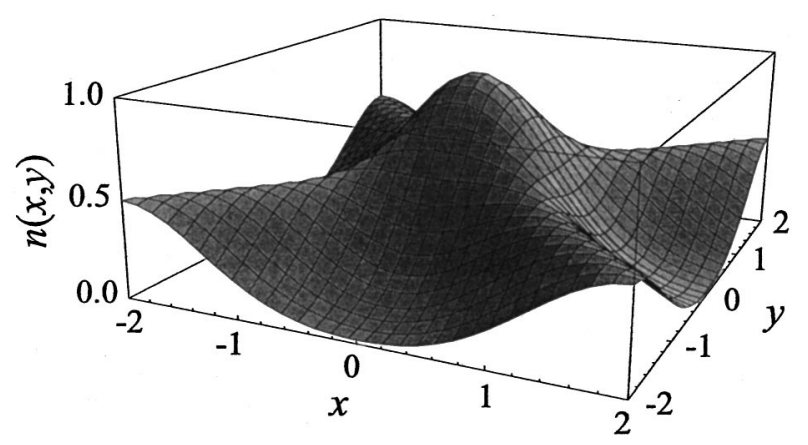

Fig. 1. Typical 2D density distribution, $n(x, y)$, derived from the condition that $\mathbf{F}_{\mathrm{abs}}$ is conservative, with $n(x, 0)=\exp \left(-x^{2}\right)$ and $\mathcal{I}=1$. Along the $|x|=|y|$ arms the density tends to the value $n(0,0) / 2=0.5$. 
been checked for consistency with Eq. (6). To solve Eq. (6) one must choose a $1 \mathrm{D}$ distribution $n(x)$ to generate the two-dimensional (2D) solution $n(x, y)$. Given a constant temperature and the density at the origin $n(0,0)$, one can make a suitable choice by including a spring force in the equations of Refs. 21 and 24. Taylor expansions for the resulting $n(x)$ profiles satisfy Eq. (6) in one dimension. They have a finite radius of convergence, but most of the spatial extent of the MOT is within this radius. Numerical integrations show that $n(x)$ is physically reasonable outside the radius of convergence of the Taylor expansion.

After the Taylor series density distribution $n(x, y)$ with conservative $\mathbf{F}_{\text {abs }}$ is generated, it can be substituted into Eq. (6). Because of the complex dependence [Eq. (3)] of the reradiation force $\mathbf{F}_{\text {rerad }}$ on the density distribution, this force has to be omitted. It is then found that the two sides of Eq. (6) are not equal, even at the low-order Taylor term level. Therefore it appears that density distributions that satisfy the conservative $\mathbf{F}_{\text {abs }}$ condition do not satisfy Eq. (6). Because the discrepancy occurs for low orders in the Taylor series, this is a more versatile proof than that which showed that the $n$ solutions were unphysical, as it does not require the series to have a relatively large radius of convergence, although the omission of $\mathbf{F}_{\text {rerad }}$ makes the result less general.

Thus we have shown that physically reasonable solutions to the WSW model equation (6) do not appear to exist on inclusion of the nonconservative absorptive force, $\mathbf{F}_{\text {abs }}$. Including $\mathbf{F}_{\text {abs }}$ may in fact make solutions impossible. This suggests that a nonconservative total force $\mathbf{F}_{\text {tot }}$ in the WSW model places restrictions on the density distribution that make it unphysical or even nonexistent.

\section{Varying-Temperature Solutions}

Having concluded that density distributions that satisfy Eq. (6) are unlikely to exist if $\mathbf{F}_{\mathrm{abs}}$ is included, we need now to suggest practicable alternatives for modeling the MOT distribution. It is possible that the assumptions of weak absorption and single photon scattering could contribute to the inconsistencies in the model. However, both assumptions should hold in the limit of low density. Because solutions do not appear to exist even in this limit, we must conclude that the limitation is with Eq. (6) itself. One obvious possible problem with the derivation of Eq. (6) is the assumption that the temperature is constant. In this section we therefore consider the possibility that the MOT temperature varies spatially.

To find the density distribution in the varyingtemperature regime we return to Eq. (5). Taking the curl of both sides leads to the condition on $n$ that

$$
\boldsymbol{\nabla} \times\left[\left(\mathbf{F}_{\text {spring }}+\mathbf{F}_{\text {abs }}+\mathbf{F}_{\text {rerad }}\right) n(\mathbf{r})\right]=\mathbf{0} .
$$

This new restriction is more complex than before, as it involves all the forces, not just $\mathbf{F}_{\mathrm{abs}}$. In the constanttemperature regime the condition $\boldsymbol{\nabla} \times \mathbf{F}_{\mathrm{abs}}=\mathbf{0}$ was a necessary, but not sufficient, condition for a solution to Eq. (6). Equation (13), however, is a necessary and sufficient condition for an $n$ solution to Eq. (5). Although solutions will now be possible, the question remains as to whether they are physically reasonable. For this reason solutions to Eq. (13) are investigated below, again by use of a 2D Taylor expansion technique in the $z=0$ plane.
Only the absorptive and spring forces, $\mathbf{F}_{\text {abs }}$ and $\mathbf{F}_{\text {spring }}$, are included in the theory, however, as it is difficult to derive a Taylor expansion for $\mathbf{F}_{\text {rerad }}$.

For simplicity the intensities of the laser beams in the $x, y$, and $z$ directions were assumed to be uniform and equal. The Taylor coefficients for the solution to Eq. (13) were found symbolically with the Mathematica computer program but have been omitted for brevity. The main feature of the Taylor coefficients for $n$ is that they depend only on the parameter ratio $3 k_{\text {spring }} /-k_{\text {abs }} I_{\text {tot }}$, and, as in the constant-temperature case, $n(x, y)$ can be found from $n(x, 0)$ [or $n(y, 0)]$. The parameter ratio should be compared with the expression for $n_{\max }$ that is discussed in Subsection 2.D. A negative value indicates that the result of the absorption and reradiation forces is compressive (as $\mathbf{F}_{\text {rerad }}$ has been omitted here). This influences the shape of the temperature distribution.

Once the Taylor series for $n(x, y)$ has been found, the corresponding series for the absorption force $\mathbf{F}_{\text {abs }}$ can be derived from Eq. (2). The pressure variation $P(x, y)$ in the trap can then be computed from these Taylor expansions by integration of Eq. (5), although an arbitrary integration constant remains. Because $n(x, 0)$ can be used to find $P(x, 0)$, the integration constant can be chosen to ensure that $P(x, 0)$ tends to zero at large $x$ values. The temperature distribution can then be found from the ideal gas law.

Thus to obtain a complete solution for $T(x, y)$ and $n(x, y)$ we must first specify the functional form of the density along the $x$ (or $y$ ) axis. Density distributions of the form $n(x, 0)=n_{0} \exp \left(-k_{\text {spring }} x^{2}\right)$ (i.e., the TL density) were used to generate the results shown here, as this distribution has simple Taylor coefficients with an infinite radius of convergence along the $x$ axis. Figure 2 shows typical results for the density and temperature distributions.

Figure 2 shows that solutions to Eq. (5) exist, at least within a finite domain, when the absorptive force is present, and that the solutions are approximately cylindrically symmetric. The divergence problem seen in the temperature plot is due to the proximity of the Taylor series's radius of convergence. This hypothesis is supported by the 1D Taylor series along the $y=x$ line. The sequence of the ratio of successive coefficients is approximately periodic, with an average absolute value of $\sim 1 /$ $(200 \mu \mathrm{m})$, so the series can be expected to converge only up to $x \approx 200 \mu \mathrm{m}$ (D'Alembert ratio test). This is also consistent with the Cauchy root test.

Having verified the existence of 2D Taylor series solutions to Eq. (5) for $n(x, y)$ and $P(x, y)$, we must check whether these solutions are physically reasonable, i.e., whether both density and pressure tend to zero at large $r$. Solutions for $n$ and $P$ with various values of $3 k_{\text {spring }} /-k_{\text {abs }} I_{\text {tot }}$ were investigated, and behavior similar to that shown in Fig. 2 was found. So, although the finite convergence domain of the $n$ and $P$ distributions makes it impossible to state categorically that they are physically reasonable, the solutions are far more promising than the constant-temperature solutions. If physical solutions do not exist with the given Gaussian initial data curves, it is still possible that they would result from other $n(x, 0)$ functions. Thus we can state that there are no physical 
solutions to Eq. (6) but that there are probably physical solutions to Eq. (5). We therefore believe that, in the regime discussed here, the temperature in a MOT must vary with position.

\section{Spherically Symmetric Distributions}

In the previous sections it became clear that physical solutions to the constant-temperature WSW equations of equilibrium [Eq. (6)] in a MOT are impossible to find. We now wish to consider approximations that can be made to the WSW model to obtain solutions, as well as solutions that are simple and essentially one dimensional.

From the relations $\boldsymbol{\nabla} \cdot \mathbf{F}_{\mathrm{abs}}=-k_{\mathrm{abs}} I_{\mathrm{tot}} n(\mathbf{r})$ and $\boldsymbol{\nabla}$ - $\mathbf{F}_{\text {rerad }}=k_{\text {rerad }} I_{\text {tot }} n(\mathbf{r})$ it is clear that $\mathbf{F}_{\text {abs }}$ and $\mathbf{F}_{\text {rerad }}$ have quite similar effects-except in opposite directions. An approximation (previously used by Steane et al. ${ }^{18}$ and Hoffman et $a l .{ }^{23}$ ) for $\mathbf{F}_{\mathrm{abs}}$ can thus be given by

$$
\mathbf{F}_{\mathrm{abs}} \approx-\frac{k_{\mathrm{abs}}}{k_{\mathrm{rerad}}} \mathbf{F}_{\mathrm{rerad}}=-\frac{\sigma_{L}}{\sigma_{R}} \mathbf{F}_{\mathrm{rerad}}
$$

This approximation preserves the essential characteristics of the absorptive force as well as allowing the total force on an atom to be conservative.

With the absorptive force effectively eliminated, only the trapping and modified reradiation forces remain, and the solution has spherical symmetry if we assume that $\mathbf{F}_{\text {spring }}=-k_{\text {spring }} \mathbf{r}$. This assumption is necessary to ren-
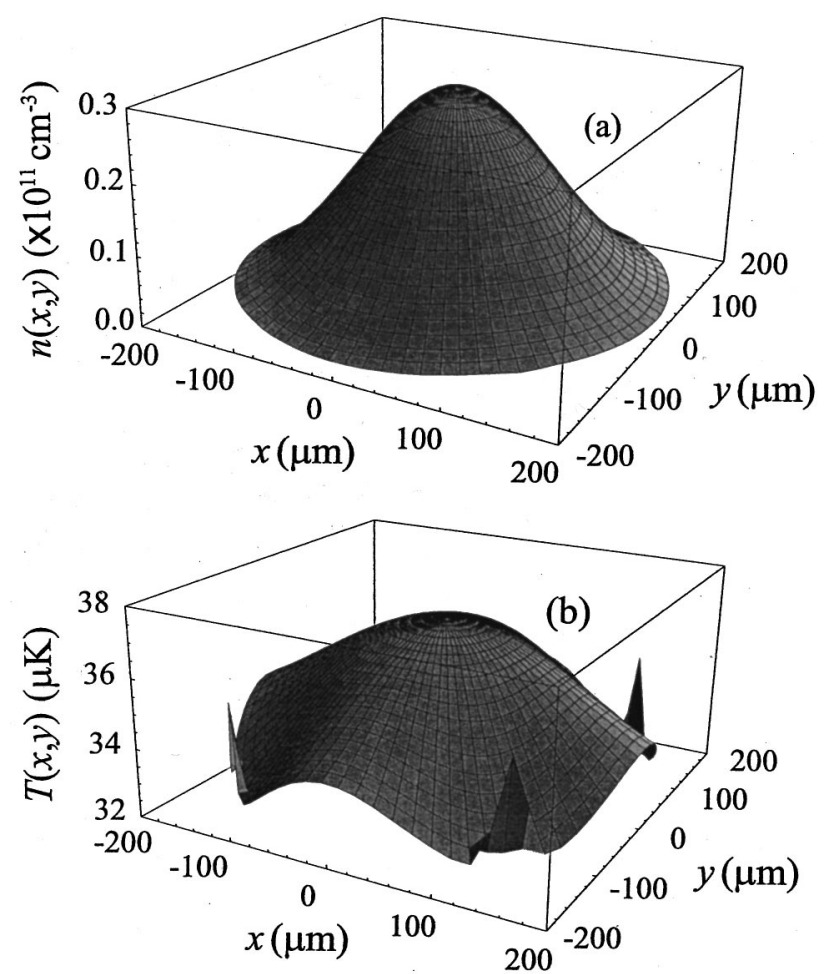

Fig. 2. Typical (a) density and (b) temperature distributions in the variable-temperature regime. The pressure distribution has a similar shape to the density. The distributions were generated from the $1 \mathrm{D}$ distribution $n(x, 0)=0.3$ $\times 10^{11} \exp \left(-k_{\text {spring }} x^{2} / 2 k_{B} T_{0}\right) \mathrm{cm}^{-3}$ by use of $k_{\text {spring }}=5$ $\times 10^{-20} \mathrm{Nm}^{-1}$ and $3 k_{\text {spring }} /-k_{\text {abs }} I_{\text {tot }}=-10^{11} \mathrm{~cm}^{-3}$. der the complicated reradiation force tractable. The total force on the atomic density distribution $n(r)$ [cf. Eq. (4)] can now be written as

$$
F_{\text {tot }}(r)=-k_{\text {spring }} r+\left(k_{\text {rerad }}-k_{\text {abs }}\right) I_{\text {tot }} \frac{\int_{0}^{r} n(u) u^{2} \mathrm{~d} u}{r^{2}} .
$$

This force depends on the difference $k_{\text {rerad }}-k_{\text {abs }}$ (i.e., $\sigma_{R}-\sigma_{L}$ ), which is (unfortunately) positive in most experimental circumstances, ${ }^{10,12}$ giving a resultant expansion force on the trap. A variety of techniques for calculating $\sigma_{L}$ and $\sigma_{R}$ have been published ${ }^{10,12,18,22}$ and will not be discussed here.

For spherically symmetric forces, Eq. (5) reduces to

$$
\begin{aligned}
\frac{\mathrm{d} P(r)}{\mathrm{d} r}= & k_{B} \frac{\mathrm{d}[n(r) T(r)]}{\mathrm{d} r} \\
= & {\left[-k_{\text {spring }} r+\left(k_{\text {rerad }}-k_{\text {abs }}\right)\right.} \\
& \left.\times I_{\text {tot }} \frac{\left.\int_{0}^{r} n(u) u^{2} \mathrm{~d} u\right]}{r^{2}}\right] n(r) .
\end{aligned}
$$

Given any function $n(r)$ for the atomic number density in the MOT, one can find the corresponding temperature distribution by following a process similar to that described above. Alternatively, if the temperature is assumed constant at $T_{0}$, one can obtain an equation for the density distribution by rearranging and differentiating Eq. (16) to give

$$
\begin{aligned}
\frac{k_{B} T_{0}}{3 k_{\text {spring }}}\left\{\frac{n^{\prime \prime}(r)}{n(r)}-\frac{1}{n^{2}(r)}\left[n^{\prime}(r)\right]^{2}\right. & \left.+\frac{2}{r n(r)} n^{\prime}(r)\right\} \\
& =-1+\frac{n(r)}{n_{\max }},
\end{aligned}
$$

where $n_{\text {max }}=3 k_{\text {spring }} /\left[\left(k_{\text {rerad }}-k_{\text {abs }}\right) I_{\text {tot }}\right]$, with the initial condition $n^{\prime}(0)=0$. Note that this equation is equivalent to the radial part of Eq. (7) expressed in spherical polar coordinates. In the constant-temperature regime it is necessary to specify a temperature $T_{0}$ and a central density $n(0)$ to obtain a unique solution for $n(r)$. In contrast, for the variable-temperature case $n(r)$ must be specified for all $r$ but the temperature $T(r)$ is determined without requiring $T(0)$.

It is already well known ${ }^{12}$ that solutions to Eq. (17) exist only when $n(0)<n_{\max }$, and the parameter $n_{\max }$ is frequently used to describe the limiting density of MOT's. ${ }^{10,12,18,20,25,26}$ As $n(0)$ approaches $n_{\max }$ in Eq. (17), the density distribution ceases to be Gaussian and increases in width: More atoms enter the trap, while the maximum density remains constant at $n_{\max }$. Figure 3 shows a sequence of three density distributions for increasing $n(0)$, illustrating the deformation of the distributions relative to the Gaussian distributions of the temperature-limited ${ }^{10}$ regime. 
As $T_{0}$ increases, a larger number of atoms is required before the density reaches the flat regime seen in Fig. 3. The shape of the trap is determined by the parameter $\eta$ $=n(0) / n_{\max }$, and the radial dimension of the MOT for fixed $\eta$ scales directly as $\sqrt{T_{0} / k_{\text {spring }}}$. The maximum possible density $n_{\max }$ is independent of $N$ and $T_{0}$; however, $n$ (0) depends on $N$ and $T_{0}$, which strongly depend on the parameters of the trap. ${ }^{27}$ So $N$ and $T_{0}$ affect $\eta$ and thus the shape of the MOT.

However if the temperature is no longer assumed to be constant in the trap, solutions cease to be restricted by the $n(0)<n_{\max }$ limit. In this case it is possible for the density to be Gaussian (or any shape), and the temperature adjusts accordingly-even if $n(0)$ approaches or exceeds $n_{\max }$. Figure 4 shows the pressure and temperature distributions that correspond to Gaussian TL density distributions for increasing $n(0)$. Under these circum-

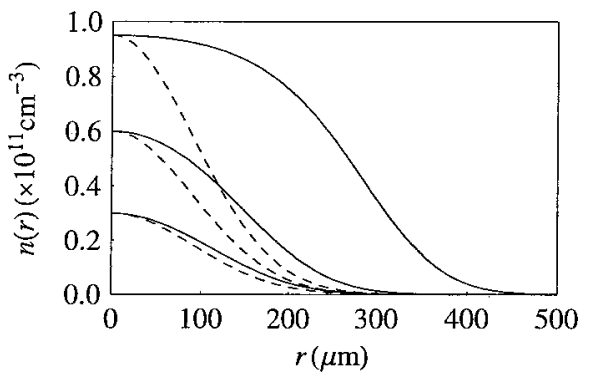

Fig. 3. Spherically symmetric density distributions (solid curves) at constant temperature $T_{0}=30 \mu \mathrm{K}$ with $n_{\max }$ $=3 k_{\text {spring }} /\left(k_{\text {rerad }}-k_{\text {abs }}\right) I_{\text {tot }}=10^{11} \mathrm{~cm}^{-3}, \quad k_{\text {spring }}=5 \times 10^{-20}$ $\mathrm{Nm}^{-1}$ for $n(0)=0.3 \times 10^{11}, n(0)=0.6 \times 10^{11}$, and $n(0)$ $=0.9510^{11} \mathrm{~cm}^{-3}$. The corresponding atom numbers are $N$ $=0.4810^{6}, N=1.5 \times 10^{6}$, and $N=9.7 \times 10^{6}$, respectively. Also shown are corresponding distributions in the TL regime (dashed curves).
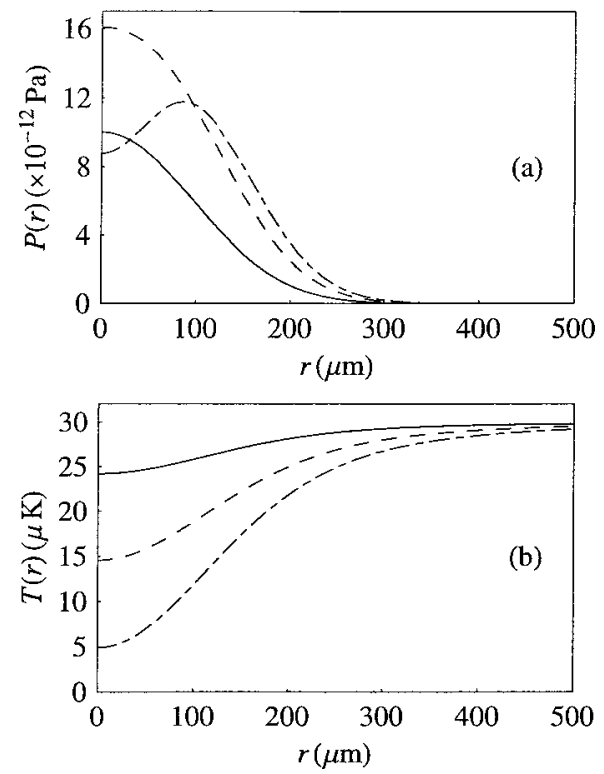

Fig. 4. Typical spherically symmetric (a) pressure and (b) temperature for distributions corresponding to a Gaussian density profile given by $n(r)=n(0) \exp \left(-k_{\text {spring }} r^{2} / 2 k_{B} T_{0}\right)$ with $k_{\text {spring }}$, $T_{0}$, and $n_{\max }$ as in Fig. 3. The density maxima were $n(0)$ $=0.3 \times 10^{11}$ (solid curves), $n(0)=0.8 \times 10^{11}$ (dashed curves), $n(0)=1.3 \times 10^{11} \mathrm{~cm}^{-3}$ (dashed-dotted curves). stances the temperature distributions are relatively constant unless the central density approaches $n_{\max }$, in which case the temperature starts to increase radially. For large values of $\eta$, less spatial variation of the temperature is observed if Gaussian profiles with larger widths are used.

It is useful to substitute the spherically symmetric constant-temperature solutions back into Eq. (5) to find the extent to which the approximations discussed in this section are valid. An estimate of the variation in $T$ for the spherically symmetric $n(r)$ at an assumed constant temperature $T_{0}$ is $|\nabla T|=\left|\mathbf{F}_{\text {tot }} / k_{B}-T_{0} \boldsymbol{\nabla}[\ln n(\mathbf{r})]\right|$. For a constant temperature the two terms in $|\nabla T|$ must be equal, so one can find an estimate of the relative variation $(\mathrm{RV})$ in the temperature by normalizing the difference, i.e., $|\nabla T|$, to the larger of the two terms:

$$
R V=\frac{\left|\mathbf{F}_{\text {tot }} / k_{B}-T_{0} \boldsymbol{\nabla}[\ln n(\mathbf{r})]\right|}{\operatorname{Max}\left\{\left|\mathbf{F}_{\text {tot }}\right| / k_{B},\left|T_{0} \boldsymbol{\nabla}[\ln n(\mathbf{r})]\right|\right\}} .
$$

With the parameters of Fig. 3, with $\sigma_{R} / \sigma_{L}=1.4$ (cf. Ref. 12) and $n(0)=6 \times 10^{10}$, the largest relative variation in the temperature is approximately $30 \%$ near the edge of the density distribution. Thus the constant-temperature approximation in the spherically symmetric solutions breaks down to some extent but only in regions of reducing atomic density.

We are not aware of any conclusive experimental observations of spatial temperature distributions within the volume of a MOT. Thus there is no direct experimental evidence to distinguish between the constant- and varying-temperature theories, although a spatially varying temperature was suggested by Sesko et al. ${ }^{12}$ to explain their observed time-of-flight spectra. In contrast, several measurements of density distributions have been reported. The observed densities have generally been independent of the number of trapped atoms, ${ }^{9,10,12,25,26,28}$ as expected if the density is limited by $n_{\max }$. However, the form of the distribution has not been as definite. Townsend et al. ${ }^{10}$ observed Gaussian density distributions with density maxima that did not show the expected dependence on the detuning and Rabi frequency, whereas Gabbanini et al. ${ }^{26}$ also observed Gaussian profiles but the maximum density behaved as expected. Several other reports of density profiles ${ }^{9,12,25,28}$ have shown flat-topped distributions similar to those in Fig. 3. In particular, recent experiments at high laser intensity ${ }^{28}$ yielded density distributions with good agreement to the approximate density functions used by Hoffmann et $a l .{ }^{23}$ to parameterize flat-topped distributions. The variety of observed distributions could be attributed to a large transitional region between the TL and multiple-scattering regimes. ${ }^{26}$ An alternative explanation that we propose based on the calculations outlined here is that the temperature may vary within the trapped atom cloud in the MOT.

Several experiments ${ }^{9,12,25,28}$ have shown that the temperature in a MOT increases with the number of trapped atoms. This temperature increase has been explained $^{20,22,25,29}$ in terms of the increased momentum diffusion caused by multiple scattering of photons. These theories predict that the temperature depends on $N^{1 / 3}$ if the density remains constant as the number of at- 
oms increases. Decreased damping and diffusion effects would be strongest at the edge of the atom cloud, and hence a radially varying temperature within the MOT could be expected. Our model predicts such a spatial variation of temperature without including the added diffusion effects directly but by assuming a Gaussian density distribution and solving for the temperature from the force equations.

\section{RING STRUCTURES}

In 1990 Walker et al. ${ }^{13}$ investigated the various structures formed in a MOT when the laser beam alignment creates a torque on the atoms. These structures are of interest because spatial distributions in a MOT may have applications in atomic light pressure lithography. ${ }^{30}$ As well as the typical ellipsoidal distribution of atoms in a MOT, Walker et al. noticed a ring structure or a rotating clump of atoms that developed around a central atomic core. This effect was explained by use of the reradiation force, and a more detailed theory was described in a subsequent paper by the same authors. ${ }^{12}$ Other groups observed rings without central cores ${ }^{14}$ and double-ring structures. ${ }^{16}$

In this section we attempt to improve the theory used in the description of these structures, as the models that are currently used are often applied outside their domains of validity. Some finer details will also be applied to the theory. The notation used here is similar to that of Bagnato et al., ${ }^{14}$ and the Rabi frequency $\Omega$ is defined by $I / I_{S}=2 \Omega^{2} / \Gamma^{2}$, where $I_{S}$ is the saturation intensity and $\Gamma$ is the natural width of the transition.

\section{A. Forces in the Ring Geometry}

To apply a torque on the atoms, we create a beam misalignment in the horizontal $(x y)$ plane is as shown in Fig. 5. We use the definition of Bagnato et al. ${ }^{14}$ and de Araujo et $a l .{ }^{16}$ for the width $w$ of the Gaussian laser beam profiles: Intensity profiles have the form $I$ $=I_{0} \exp \left(-\rho^{2} / w^{2}\right)$, where $\rho$ is the radial distance from the laser beam's axis of propagation. This definition is different from that of the waist often used in laser physics (and in Ref. 15).

Various methods have been used to predict atomic orbits in the MOT geometry of Fig. 5. Probably the simplest technique adopted is to consider a radial line in the $x y$ plane (e.g., the $x$ axis) and compare the variation of the trapping forces acting on an atom located along this line with the required centripetal force. ${ }^{14,17}$ By finding a point where the trapping force is equal to the centripetal force and requiring that these forces obey stability criteria in the vicinity of that point, one can find the equilibrium radii at which atoms orbit. An alternative method is to use the $2 \mathrm{D}$ time-averaged position-dependent forces acting on the atoms in the $x y$ plane and solve for the stable atomic orbits numerically. ${ }^{12,14,16,17}$ This is also a useful test for the assumption of cylindrical symmetry in the $1 \mathrm{D}$ model discussed above.

We discuss refinements to the existing $1 \mathrm{D}$ models and compare our results with a full 3D Monte Carlo simulation. The advantage of such a simulation compared with previous models is that diffusion can also be included in the model, providing information about the 3D velocity and spatial distributions in a MOT as well as about the coupling between the various structures (rings and cores) within a MOT. We begin with a discussion of the $1 \mathrm{D}$ model.

As the average force on the atoms is in the $z=0$ plane, only the average forces applied by the $x$ and $y$ beams need be considered. Because $v_{x}=0$ for radially stable orbits crossing the $x$ axis, the total trapping force in the $-x$ direction (which is inward for $x>0$ ) can be written as

$$
\begin{aligned}
F_{\text {trap }}= & \frac{\hbar k_{L} \Omega_{0}^{2}}{\Gamma e^{s^{2} / w^{2}}}\left[\frac{1}{1+\frac{I_{\text {tot }}(x)}{I_{S}}+\left(\frac{2}{\Gamma}\right)^{2}\left(\Delta+\omega_{B} b x\right)^{2}}\right. \\
& \left.-\frac{1}{1+\frac{I_{\text {tot }}(x)}{I_{S}}+\left(\frac{2}{\Gamma}\right)^{2}\left(\Delta-\omega_{B} b x\right)^{2}}\right],
\end{aligned}
$$

where $\omega_{B}=\mu_{B} / \hbar, \Delta$ is the laser detuning, $b$ is the magnetic field gradient, and $\Omega_{0}$ is the on-axis laser Rabi frequency. The $\exp \left(-s^{2} / w^{2}\right)$ factor occurs because the laser beam axes are offset from the $x$ axis. Here we are neglecting the absorption and reradiation forces to find an analytical model for the ring radius.

Because of the varying-intensity imbalance of the $y$ beams along the $x$ axis, the atoms will also experience forces in the $y$ direction that depend on their $x$ position. The average force on an atom on the $x$ axis that is due to the laser beams traveling in the $\pm y$ directions is

$$
\begin{gathered}
F_{y}=\frac{\hbar k_{L} \Omega_{0}^{2}}{\Gamma}\left\{\frac{\exp \left[-(x+s)^{2} / w^{2}\right]}{1+\frac{I_{\text {tot }}(x)}{I_{S}}+\left(\frac{2}{\Gamma}\right)^{2}\left(\Delta-k_{L} v_{y}\right)^{2}}\right. \\
\left.-\frac{\exp \left[-(x-s)^{2} / w^{2}\right]}{1+\frac{I_{\text {tot }}(x)}{I_{S}}+\left(\frac{2}{\Gamma}\right)^{2}\left(\Delta+k_{L} v_{y}\right)^{2}}\right\}
\end{gathered}
$$

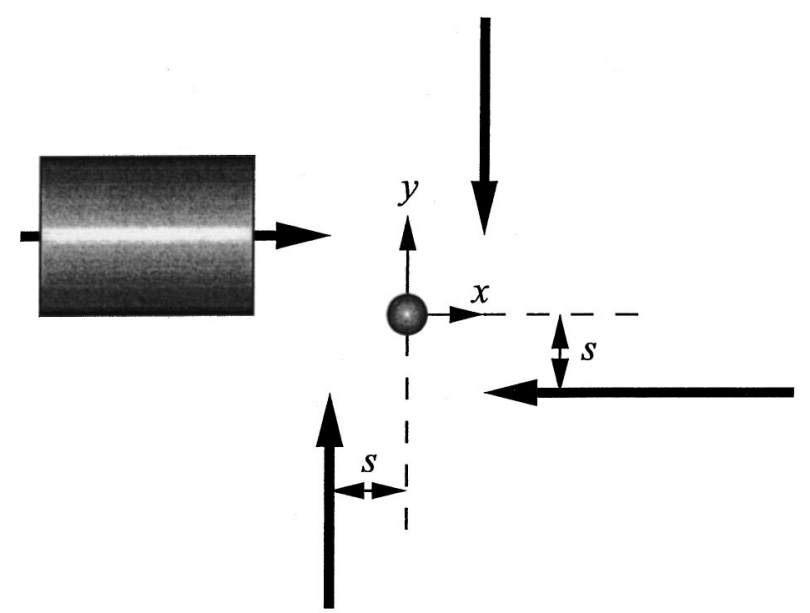

Fig. 5. Racetrack beam alignment typically used to create orbital modes in a MOT. The bold arrows indicate the axes and propagation directions of the four Gaussian laser beams in the $z=0$ plane. The $z$ beams necessary for confinement in the $z$ direction are not shown. 
For an atom to move in a stable orbit, the $y$ component $F_{y}$ of the force must be zero and the $x$ component $F_{\text {trap }}$ must provide the required centripetal force, $F_{\text {cent }}$ $=m v_{y}^{2} / x$, to keep the atom moving on a circular path of radius $x$ at constant speed $v_{y}$. An additional requirement for the orbit to be stable is that the trapping force must be stronger than the required centripetal force just outside the orbit radius and weaker just inside the orbit.

Bagnato et $a l .{ }^{14}$ and Guedes et al. ${ }^{15}$ used linear approximations for both the trapping force and the force in the $y$ direction. They then obtained the equilibrium $y$ velocity and used a graphic technique to compare trapping and centripetal forces and to establish the existence of stable orbits. However, it is possible to find the equilibrium velocity from Eq. (20) without using the linear approximation. One can then use this equilibrium $y$ velocity to compare the trapping force with the centripetal force and thus to find the radii of stable orbits. We show below that the linear approximations for the two forces can cause significant errors in the calculated orbit radius.

The two 1D theories described above will now be compared with a 3D Doppler Monte Carlo (MC) simulation of velocity and position distributions in a MOT. The MC models the position and velocity of atoms in the MOT based on randomized momentum-conserving photon absorptions from the six laser beams weighted with each atom's full 3D position and velocity-dependent relative absorption rates from these beams. The absorption rates include the variation of the intensity in the Gaussian beams, the variation of light polarization, and the saturation, Doppler, and Zeeman terms in the transition rates. An isotropic spontaneous emission follows each absorption process, enhancing the diffusion in the model. An $F=0 \rightarrow F^{\prime}=1$ atomic transition is used for simplicity and to provide more-direct comparison with previous calculations.

Figure 6 shows the difference between the trapping and centripetal forces predicted by both the linear and the nonlinear force equations. It is worth noting that the $z$ laser beams may well require different waist sizes and intensities from the $x y$ beams to provide $z$ confinement for atoms orbiting at large radii. It can be seen that both models predict the formation of a ring for these parameter values but that the expected radii are markedly different. Figure 7 shows the results of the MC simulation for the same physical trap parameters. It can be seen that the ring radius predicted by the linear equations is substantially different from the results of the MC simulation. The more exact $1 \mathrm{D}$ nonlinear equations developed here give better agreement but still have a small error. This error is due to the assumption in deriving Eq. (20) that atoms on the $x$ axis are subject to purely $\pi$-polarized $y$ lasers. If the correct polarization is included, Eq. (20) must be solved numerically. The forces then take on a form similar to that shown in Fig. 6, and the ring radius predicted exactly matches the radius shown in Fig. 7 .

We can see the problems with the linear theory more clearly by comparing the plots of $F_{\text {cent }}(x)$ and $F_{\text {trap }}(x)$ for the two theories in Fig. 8. The dashed curves correspond to the linear equations described above, and the solid curves correspond to the more-exact versions of $F_{\text {cent }}$ and $F_{\text {trap }}$. The difference between the two approximations

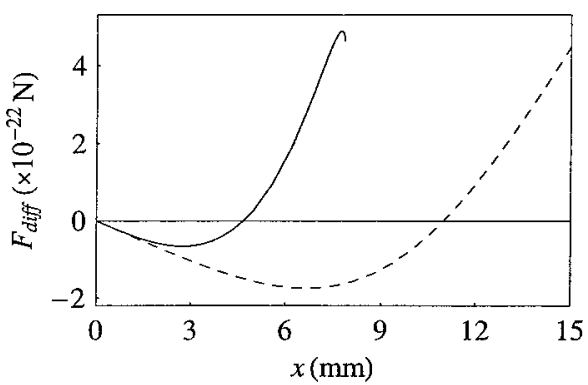

Fig. 6. Difference, $F_{\text {diff }}(x)=F_{\text {trap }}(x)-F_{\text {cent }}(x)$, between the trapping and the centripetal forces predicted by the nonlinear (solid curve) and the linear (dashed curve) theories for sodium atoms; $b=15 \mathrm{G} / \mathrm{cm}, \quad \Delta=-2 \Gamma, \quad w=1 \mathrm{~cm}, \quad s=1.2 \mathrm{~cm}, \quad \Omega_{0}$ $=\sqrt{0.15} \Gamma$. The linear theory predicts that a ring with a radius of $11 \mathrm{~mm}$ will be produced, whereas the more-exact theory predicts a radius of $4.6 \mathrm{~mm}$.

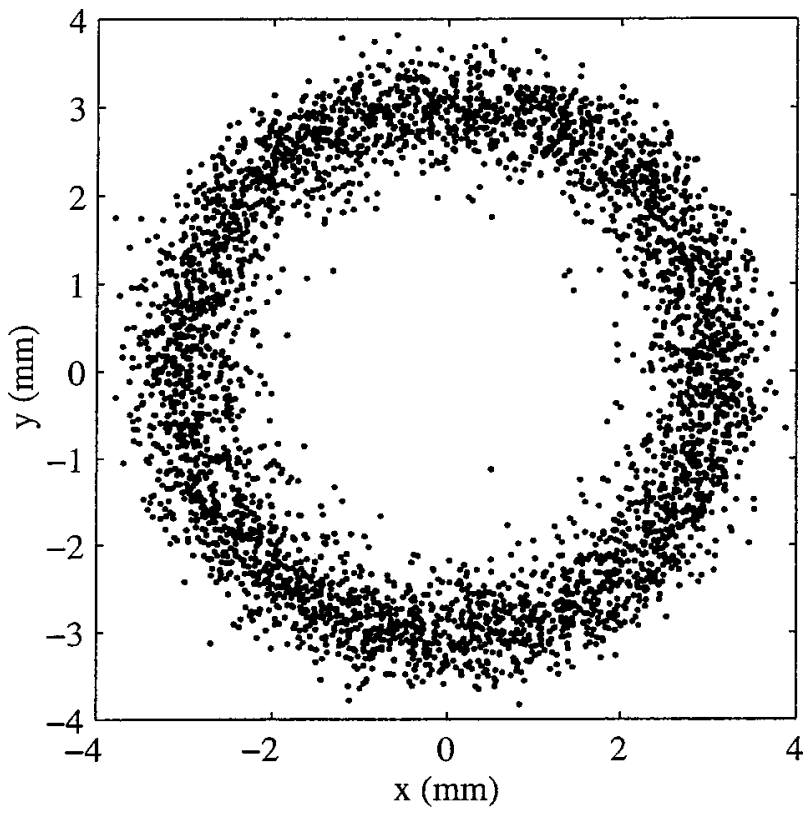

Fig. 7. Results of a MC simulation of a MOT under the same conditions as for Fig. 6. The simulation models 100 atoms released at the origin of a MOT and then allowed to reach equilibrium. The image is a conglomerate of snapshots from various times, where each dot represents an atom. The ring radius is $r \approx 3 \mathrm{~mm}$.

for $F_{\text {cent }}$ at large $x$ is substantial for most values of $s$. In fact, the more-exact calculation of $F_{\text {cent }}$ shows that there are often regions where the intensity imbalance is so high that no equilibrium velocity exists-atoms will accelerate out of the MOT. This is shown in Fig. 8 by the limited extent of $F_{\text {cent }}$ in the full calculation. The bell shape of the simplified expression for $F_{\text {cent }}$ was crucial to finding rings with central balls in Ref. 14. However, the full expression for $F_{\text {cent }}$ is approximately linear and of finite extent and therefore does not predict this type of trapped cloud.

\section{B. Importance of Reradiation}

The forms of the more-exact trapping and centripetal forces imply that it will be difficult, if not impossible, to obtain a situation in which a ring will form with an inner 
core (or ring) of atoms, i.e., a regime where the difference $F_{\text {trap }}(x)-F_{\text {cent }}(x)$ has positive slope near zero crossings at two distinct $x$ values. Whereas it is relatively easy to obtain laser configurations that give ring-shaped distributions without central cores by use of either the 1D theory or the MC simulation, it is likely that the present Doppler theory alone will not permit such distributions without the linear approximations described above. However, this type of structure has been observed experimentally, ${ }^{12-14,16}$ so it remains to find additions to the theory to explain their formation. It is unlikely that a sub-Doppler model ${ }^{18}$ is required because of the high intensities, and intensity imbalances, required in the experiments. The most likely possibility, suggested by Sesko et al., ${ }^{12}$ is that the reradiation force causes the ring with the core to form.

Guedes et al. ${ }^{15}$ showed that, for small numbers of atoms $\left(N<10^{7}\right)$, it is possible experimentally to obtain rings with radii that do not depend on $N$ (and hence on reradiation). However, rings with large numbers of atoms required the reradiation force for a complete explanation. ${ }^{15,30}$ The theory used to explain the experimentally observed radii at low $N$ was similar to that in Ref. 14 and showed reasonable agreement with experiment, suggesting that Doppler theory is quite adequate to describe a ring without a central core in the low- $N$ regime. It is therefore possible to explain rings without reradiation, but a similar quantitative test has not been done for the ring-with-core configuration.

Bagnato et al. ${ }^{14}$ attempted to show that the formation of rings, both with and without central cores, could be explained in the absence of reradiation. However, high intensities and small detunings were necessary to produce the ring-and-core configuration in both theory and experiment, which suggests that this configuration occurs when the reradiation force is relatively large. In fact, the theoretical parameters that are necessary to explain the observed ring-and-core and double-ring configurations are often quite different from the experimental parameters. ${ }^{14,16}$

Another point that suggests that the ring-and-core configuration requires the presence of the reradiation force is that the theory used both here and in Refs. 14 and 16 becomes inaccurate at high intensities. High intensities are needed to generate the ring-and-core configuration, but the equations should be used only when $2 \Omega_{0}{ }^{2} / \Gamma^{2}$ $\approx 1$. $^{3,31}$ In Refs. 14 and 16 this parameter often needs to

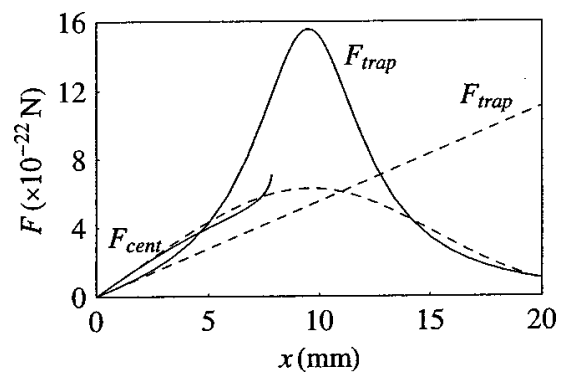

Fig. 8. Comparison of the nonlinear (solid curves) and the linear (dashed curves) calculations of the trapping force $F_{\text {trap }}(x)$ and the required centripetal force $F_{\text {cent }}(x)$. The linear and the nonlinear models for these forces agree at small $x$ values. The same trap parameters are used as in Figs. 6 and 7. be set to values of 288 or more to yield a theoretical ringand-core distribution. Even if the theory were valid at such high intensities, the reradiation force would certainly be evident and need to be considered in the description.

Similarly, de Araujo et $a l .{ }^{16}$ used a theoretical model with $2 \Omega_{0}{ }^{2} / \Gamma^{2}=338$ to explain the double-ring structure that was experimentally observed at high intensity and large beam misalignment. Their comparison of $F_{\text {trap }}$ and $F_{\text {cent }}$ implied that an outer ring should form but that atoms would be only weakly held in the inner ring. With such a weak force holding the atoms in the inner orbit, if diffusion were included in the model it would be highly likely that atoms would boil off from the internal ring. The internal ring would therefore have a much lower population than the external ring (which is not experimentally observed). It is the opinion of the present authors that ring-and-core and double-ring configurations (when $2 \Omega_{0}^{2} / \Gamma^{2}<1$ ) can be explained only if the reradiation force is considered.

\section{CONCLUSION}

To conclude, we initially looked at density distributions in a magneto-optical trap under the standard counterpropagating laser beam geometry. Using the differential equation describing the uniform temperature MOT [Eq. (6)], we showed that the atomic density distribution must be constrained in such a way as to produce a conservative absorption force. Density solutions consistent with this restriction can be derived by use of a Taylor series technique, with absorption and reradiation forces included in the analysis. However, we found that, although a density solution that obeys the conservative force restriction can always be found, such solutions are not physically reasonable and moreover do not satisfy Eq. (6), at even the low-order Taylor terms.

Thus we believe that the assumption of a completely uniform temperature within the trapped cloud of atoms must be abandoned. The extra degree of freedom provided by a variable temperature allows physically reasonable density and pressure distributions to be found. To support this claim, we looked for Taylor series solutions to the more general equation (5). Unfortunately, we have been able to find examples of these only by ignoring the reradiation force, and the Taylor series solutions have only a finite radius of convergence. However, these problems do not detract from the conclusion regarding the temperature variation. We then, by making an approximation that induces spherical symmetry, discussed simple distributions in both constant- and varyingtemperature cases and showed how they may explain some observations of trap temperatures.

Subsequently, by considering the average forces on an atom in the ring-shaped distributions produced in a MOT with racetrack geometry trapping beams, we calculated the radii of stable orbits along which atoms move. We avoided the linear approximations to these forces that have sometimes been used and found good agreement with a 3D Monte Carlo simulation of a MOT under these conditions. Additionally, we showed that approximations used previously may change the conditions under 
which such structures can form. Finally, the difficulty of forming ring and central core configurations under this improved treatment was used to add weight to previously published statements that the reradiation force plays a significant part in the formation of ring-with-core configurations.

\section{ACKNOWLEDGMENT}

A. Arnold was supported by a Blair postgraduate award during part of this research.

*Present e-mail address, a.arnold@phys.strath.ac.uk.

\section{REFERENCES}

1. E. L. Raab, M. Prentiss, A. Cable, S. Chu, and D. E. Pritchard, "Trapping of neutral sodium atoms with radiation pressure," Phys. Rev. Lett. 59, 2631-2634 (1987).

2. C. Monroe, W. Swann, H. Robinson, and C. Wieman, "Very cold trapped atoms in a vapor cell," Phys. Rev. Lett. 65, 1571-1574 (1990).

3. P. D. Lett, W. D. Phillips, S. L. Rolston, C. E. Tanner, R. N. Watts, and C. I. Westbrook, "Optical molasses," J. Opt. Soc. Am. B 6, 2084-2107 (1989).

4. P. D. Lett, R. N. Watts, C. I. Westbrook, W. D. Phillips, P. L. Gould, and H. J. Metcalf, "Observation of atoms laser cooled below the Doppler limit," Phys. Rev. Lett. 61, 169172 (1988).

5. J. Dalibard and C. Cohen-Tannoudji, "Laser cooling below the Doppler limit by polarization gradients: simple theoretical models," J. Opt. Soc. Am. B 6, 2023-2045 (1989).

6. P. J. Ungar, D. S. Weiss, E. Riis, and S. Chu, "Optical molasses and multilevel atoms: theory," J. Opt. Soc. Am. B 6, 2058-2071 (1989).

7. A. M. Steane and C. J. Foot, "Laser cooling below the Doppler limit in a magneto-optical trap," Europhys. Lett. 14, 231-236 (1991).

8. W. Ketterle, K. B. Davis, M. A. Joffe, A. Martin, and D. E. Pritchard, "High densities of cold atoms in a dark spontaneous-force optical trap," Phys. Rev. Lett. 70, 2253 2256 (1993)

9. M. Drewsen, P. Laurent, A. Nadir, G. Santarelli, A. Clairon, Y. Castin, D. Grison, and C. Salomon, "Investigation of subDoppler cooling effects in a cesium magneto-optical trap," Appl. Phys. B 59, 283-298 (1994).

10. C. G. Townsend, N. H. Edwards, C. J. Cooper, K. P. Zetie, C. J. Foot, A. M. Steane, P. Szriftgiser, H. Perrin, and J. Dalibard, "Phase-space density in the magneto-optical trap," Phys. Rev. A 52, 1423-1440 (1995).

11. M. H. Anderson, J. R. Ensher, M. R. Matthews, C. E. Wieman, and E. A. Cornell, "Observation of Bose-Einstein condensation in a dilute atomic vapor," Science 269, 198-201 (1995).

12. D. W. Sesko, T. G. Walker, and C. E. Wieman, "Behavior of neutral atoms in a spontaneous force trap," J. Opt. Soc. Am. B 8, 946-958 (1991).

13. T. Walker, D. Sesko, and C. Wieman, "Collective behavior of optically trapped neutral atoms," Phys. Rev. Lett. 64, 408-411 (1990).

14. V. S. Bagnato, L. G. Marcassa, M. Oria, G. I. Surdutovich, R. Vitlina, and S. C. Zilio, "Spatial distribution of atoms in a magneto-optical trap," Phys. Rev. A 48, 3771-3775 (1993).

15. I. Guedes, M. T. de Araujo, D. M. B. P. Milori, G. I. Surdutovich, V. S. Bagnato, and S. C. Zilio, "Forces acting on magneto-optically trapped atoms," J. Opt. Soc. Am. B 11, 1935-1940 (1994).

16. M. T. de Araujo, L. G. Marcassa, S. C. Zilio, and V. S. Bagnato, "Double-ring structure: another variant in the spatial distribution of cold sodium atoms," Phys. Rev. A 51, 4286-4288 (1995)

17. V. S. Bagnato, L. G. Marcassa, M. Oriá, G. I. Surdutovich, and S. C. Zilio, "Spatial distributions of optically trapped cooled neutral atoms," Laser Phys. 2, 172-177 (1992).

18. A. M. Steane, M. Chowdhury, and C. J. Foot, "Radiation force in the magneto-optical trap," J. Opt. Soc. Am. B 9, $2142-2158$ (1992).

19. M. Gajda and J. Mostowski, "Three-dimensional theory of the magneto-optical trap: Doppler cooling in the lowintensity limit," Phys. Rev. A 49, 4864-4875 (1994).

20. G. Hillenbrand, K. Burnett, and C. J. Foot, "Effect of scattered radiation on sub-Doppler cooling," Phys. Rev. A 52, 4763-4786 (1995)

21. J. Dalibard, "Laser cooling of an optically thick gas: the simplest radiation pressure trap?," Opt. Commun. 68, 203208 (1988).

22. K. Ellinger, J. Cooper, and P. Zoller, "Light-pressure force in $\mathrm{N}$-atom systems," Phys. Rev. A 49, 3909-3933 (1994).

23. D. Hoffman, P. Feng, and T. Walker, "Measurement of Rb trap-loss collision spectra," J. Opt. Soc. Am. B 11, 712-720 (1994).

24. A. P. Kazantsev, G. I. Surdutovich, D. O. Chudesnikov, and V. P. Yakovlev, "Scattering, velocity bunching, and selflocalization of atoms in a light field," J. Opt. Soc. Am. B 6, 2130-2139 (1989).

25. C. J. Cooper, G. Hillenbrand, J. Rink, C. G. Townsend, K. Zetie, and C. J. Foot, "The temperature of atoms in a magneto-optical trap," Europhys. Lett. 28, 397-402 (1994).

26. C. Gabbanini, A. Evangelista, S. Gozzini, A. Lucchesini, A. Fioretti, J. Müller, M. Colla, and E. Arimondo, "Scaling laws in magneto-optical traps," Europhys. Lett. 37, 251256 (1997).

27. K. Lindquist, M. Stephens, and C. Wieman, "Experimental and theoretical study of the vapor-cell Zeeman optical trap," Phys. Rev. A 46, 4082-4090 (1992).

28. S. Grego, M. Colla, A. Fioretti, J. H. Müller, P. Verkerk, and E. Arimondo, "A cesium magneto-optical trap for coldcollision studies," Opt. Commun. 132, 519-526 (1996).

29. G. Hillenbrand, C. J. Foot, and K. Burnett, "Heating due to long-range photon exchange interactions between cold atoms," Phys. Rev. A 50, 1479-1489 (1994).

30. M. T. de Araujo, R. J. Horowicz, D. Milori, A. Tuboy, R. Kaiser, S. C. Zilio, and V. S. Bagnato, "Time of flight technique to determine temperature of atoms in a ring-shaped trap," Opt. Commun. 119, 85-89 (1995).

31. P. L. Gould, P. D. Lett, and W. D. Phillips, "New measurements with optical molasses," in Laser Spectroscopy VIII, W. Persson and S. Svanberg, eds. (Springer-Verlag, Berlin, 1987), pp. 64-67. 